\title{
Medir y evaluar la excelencia de la investigación científica: retos y soluciones
}

\author{
Antonio Perianes Rodríguez \\ Carlos Olmeda Gómez \\ M. ${ }^{a}$ Antonia Ovalle Perandones \\ Universidad Carlos III de Madrid
}

\section{Resumen}

La calidad de la investigación es un tema de capital importancia en la ciencia moderna y ayuda a la comprensión de las diferencias que existen en la reputación de los diferentes científicos y entre las universidades y centros de investigación. La actividad científica se ha evaluado tradicionalmente mediante el uso de indicadores bibliométricos que miden las frecuencias de aparición de los artículos en las revistas punteras de una especialidad o en el número de citas que atraen. Aunque la excelencia se considera deseable, es raramente definida con el suficiente detalle como para ser medida, lo que se traduce en una búsqueda sin significado ni sentido. Con tal motivo se ha llevado una exhaustiva revisión bibliográfica para conocer los indicadores bibliométricos que de forma precisa y no ambigua describen la noción de excelencia en la investigación.

Palabras clave: Excelencia en la investigación. Indicadores de la investigación. Calidad en la investigación. Excelencia científica.

\section{Abstract}

Research quality is the cornerstone of modern science; it aids in the understanding of reputational differences among scientific and academic institutions. Traditionally, scientific activity is measured by a set of indicators and well-established bibliometric techniques based on the number of academic papers published in top-ranked journals or on the number of citations of these papers. Although research excellence is generally agreed to be desirable, it is rarely defined in enough detail to measure it, which may render the pursuit of research excellence meaningless or impractical in terms of meeting measurable targets. So that we made an exhaustive bibliographic revision of the most recent papers, in order to collect all advanced bibliometric indicators to unambiguously describe and establish research excellence.

Key words: Research excellence. Research indicators. Research quality. Scientific excellence. 


\section{Introducción (1)}

La naturaleza de la investigación que llevan a cabo los centros públicos de investigación se está modificando, y esta transformación obedece a diversos motivos. El primero de ellos es consecuencia del creciente interés por el establecimiento de objetivos dirigidos de investigación por parte de los gestores de las políticas científicas y tecnológicas, tal y como queda ejemplificado en las líneas de los programas marco de la Unión Europea, los planes nacionales de I + D o las políticas de ciencia y tecnología de los gobiernos regionales. Ello se produce en un contexto económico turbulento, con crecientes dificultades para la obtención de recursos financieros institucionales públicos, en unas actividades como las de la investigación, que son extraordinariamente dependientes de los recursos externos suministrados por el sistema político. Es bajo estas condiciones como surge la necesidad de plantear una nueva definición del control de la calidad de la investigación, donde la excelencia científica se convierte en un concepto de capital importancia para los gestores y evaluadores de las políticas de ciencia y tecnología.

El control de calidad en la investigación tiene dos componentes: uno es institucional y se refiere a la posición espacial de una actividad investigadora concreta en el paisaje cognitivo, y el otro es de carácter estrictamente cognitivo y pertenece a la organización social en la que se lleva a cabo tal investigación. De forma tradicional, en el primer caso el control es ejercido por diferentes tipos de instituciones productoras del conocimiento, cada una de las cuales tiene sus propios límites, estructuras de aprendizaje y reglas de comportamiento. Tales instituciones incluyen, por ejemplo, universidades, academias nacionales y sociedades profesionales. Cada una dispone de formas de controlar a sus miembros; algunas ofrecen formación y establecen procedimientos para producir y convalidar el conocimiento.

El segundo componente del control de calidad se relaciona con mecanismos que definen qué problemas hay que abordar, cómo se tienen que afrontar y qué resultados se consideran válidos. El éxito en las actividades investigadoras quizá pueda describirse sintéticamente como la excelencia definida por los colegas disciplinares. Así, en la forma tradicional, el control de calidad de las actividades de investigación ha sido medido por diferentes criterios; entre ellos, los juicios de revisión por parte de especialistas iguales, la contribución previa a la disciplina, el desarrollo de objetivos de investigación basados en las preocupaciones e intereses intelectuales de la disciplina o la búsqueda del consenso entre la comunidad de practicantes. Sin embargo, uno de los cambios que se perciben en la actualidad es la inclusión de criterios adicionales, como la eficiencia o utilidad de la investigación, definidas en términos de la contribución que ha hecho el trabajo a la solución de problemas transdisciplinares, el uso y el contexto de la aplicación de la investigación o la trascendencia social, económica o política de los proyectos de investigación. 


\section{Hacia una noción de excelencia científica}

Los gestores de los organismos dedicados a la investigación, las agencias de evaluación, las entidades de financiación y los gobiernos siempre se hacen la misma pregunta: ¿cómo se puede definir, reconocer y comparar la calidad del trabajo científico de la forma más objetiva posible?

Esa calidad del trabajo científico no es directamente mensurable. Existen numerosas definiciones de prestigio científico, elite científica y reputación de jerarquías en la literatura sociológica, y su significado exacto depende de la escuela de pensamiento y del contexto teórico o metodológico empleados.

Aunque es indudable que la revisión por pares tiene que seguir siendo el principal método de juicio de la calidad científica en el marco de la evaluación a posteriori, no es menos cierto que todos los juicios basados en la opinión de expertos tienen importantes inconvenientes y desventajas. Uno de ellos, quizá el principal, es la subjetividad, o lo que es lo mismo, la dependencia que tiene el resultado final respecto de la elección de los encargados de realizar esa evaluación.

A ello se unen la aparición de nuevos campos y la creciente interdisciplinariedad, que dificultan aún más la formación de opiniones válidas sobre el rendimiento de lo que se está evaluando. Por todo ello, es difícil lograr aplicaciones abiertas y claras a partir de la evaluación por pares. Sin embargo, los nuevos desarrollos en el campo de los estudios cuantitativos de la ciencia ofrecen métodos para apoyar la revisión por pares con el fin de obtener mayor objetividad y transparencia. Hoy día se acepta que los estudios bibliométricos miden, sin demasiados problemas y con un alto grado de precisión, los logros y la trayectoria científica de determinadas disciplinas, principalmente las relacionadas con la investigación básica. Si bien es cierto que este no es el único aspecto que debe ser considerado: patentes, conexiones con la industria, financiación, organización de congresos, calidad de la enseñanza, influencia cultural, reconocimiento social, premios internacionales, actividad editorial y otros factores relativos a la intensidad y creatividad de la investigación y sus resultados económicos son también muy importantes. Por tanto, el análisis bibliométrico es solo una parte, pero pone de manifiesto datos esenciales sobre los resultados de investigación que deben ser tenidos en consideración (Rovira, 2003; Tijssen, 2003).

Pero ¿es posible definir excelencia científica en un contexto de comparación institucional? La excelencia es a menudo entendida como un simple sinónimo de buena calidad. Su búsqueda es indiscutible dentro de las comunidades científicas; lograr la excelencia en el sentido de contribuir a crear progreso ha sido siempre el objetivo clave de los investigadores y los centros de investigación.

Actualmente, la excelencia ha adoptado una forma económica marcada por un énfasis en la competitividad y los centros de excelencia. La excelencia y la 
comercialización de la investigación se han convertido en los objetivos prioritarios de los gestores de política científica. Los tres conceptos más importantes de la excelencia son la creación de nuevo conocimiento científico y técnico de alta calidad, su transmisión a la comunidad de usuarios y la explotación comercial de ese conocimiento. Alcanzar y mantener la excelencia es lo que se desea tanto en el ámbito nacional como en el internacional, además de atraer suficientes recursos para mantener el liderazgo. Luchar por la excelencia, siendo excelentes, es lo primordial en política científica y está a la vanguardia de las evaluaciones del desarrollo científico sobre las que informan agencias cuyo objetivo no es otro que encontrar y promover la excelencia. Esta prioridad política se ha generalizado entre los países científicamente más avanzados, aunque existen enormes variaciones en cuanto a cómo lograrla, por un lado, y a cómo integrar acciones y decisiones políticas para su mantenimiento, por otro. Lograr la excelencia ha conseguido tal estatus que se ha convertido en uno de los objetivos prioritarios del Sexto Programa Marco de la Unión Europea.

Aunque la excelencia se considera deseable, es raramente definida con el suficiente detalle como para ser medida, lo que se traduce en una búsqueda sin significado ni sentido. En general, este concepto se refiere a sobrepasar algo o a alguien en calidad, por lo que siempre es una medida de comparación, y sus significados e implicaciones vendrán definidos por el contexto en el que sea abordado. En este caso la comparación debe centrarse en el valor añadido o la significación especial de las actividades investigadoras desarrolladas por una institución en relación con otras organizaciones con las que se compara.

La noción de excelencia parece variar de un campo a otro, de un país a otro, dependiendo del dominio científico, sus objetivos y sus políticas. Por tanto, su utilización es cada vez más vaga y confusa debido a que sus atributos interrelacionados permiten usos flexibles en muchos niveles y por muy diferentes razones. A pesar de todo, sorprende ver que este concepto, que conlleva una complicación inherente, cobra tal importancia en el debate sobre política científica y en las prácticas de gestión de la investigación. Su ambigüedad lo convierte en un término atractivo para las organizaciones científicas; sin embargo, su inmensa popularidad como tarjeta de presentación y vehículo de inestimable valor para mejorar las relaciones públicas contrasta con los escasos esfuerzos para afianzar su definición (Tijssen, 2003).

Sin duda alguna, la aproximación conceptual y operacional a un fenómeno multidimensional como la excelencia es difícil. Nos encontramos ante un concepto dinámico cuyo significado puede cambiar durante su formulación o como resultado de su medida.

El diccionario de la Real Academia de la Lengua define excelencia como "superior calidad o bondad que hace digna de singular aprecio y estimación una cosa".

Scire. 12 : 2 (jul.-dic. 2006) 87-98. ISSN 1135-3716. 
De aquí podemos extraer un significado general: excelencia como expresión comparativa que denota superioridad frente a otros en términos de calidad basada en la obtención de mejores resultados dentro de un conjunto de entidades comparables.

Por tanto, el término excelencia debe ser entendido con el significado de expresión comparativa que denota superioridad frente a otros en términos de cantidad y/o calidad. El siguiente paso debe ser el desarrollo de modelos que permitan obtener medidas comparativas y de interpretación útiles para analizar la excelencia.

\subsection{Excelencia científica y excelencia investigadora}

Una vez apuntalado el concepto de excelencia, es preciso hacer una distinción entre excelencia investigadora y excelencia científica. Las actividades de investigación son una parte del trabajo científico, aunque son difíciles de diferenciar de otras actividades relacionadas con la ciencia, como la enseñanza o el asesoramiento profesional. Como ya hemos comentado, la excelencia científica incluye aspectos cognitivos, sociales y de organización de cada grupo de investigación, lo que se conoce como capital intelectual y capital social de sus miembros, a los que se une el nivel de equipamiento disponible. También pueden considerarse desarrollos de excelencia científica actividades como el reclutamiento e instrucción de estudiantes, la comunicación de los hallazgos científicos o el éxito en la transferencia de resultados de investigación a sectores técnicos y de innovación.

El concepto de centro de excelencia investigadora, por otro lado, implica el desarrollo de investigaciones por parte de un grupo limitado de investigadores organizados dentro de una entidad física o red. Desde el punto de vista de la organización, lograr la excelencia supone contar con una infraestructura de conocimiento capaz de producir una tensión creativa entre los individuos que la conforman, su misión y sus objetivos. Por tanto, alcanzar la excelencia requiere no solo recursos e inversiones, sino también equipos e individuos con el conocimiento, las habilidades y el talento necesarios para obtener buenos resultados de investigación. Así, la excelencia investigadora puede tener también un significado orientado a la aplicación, es decir, a la generación de resultados de relevancia tecnológica, de gran valor social o que reportan importantes beneficios económicos. En estos casos la excelencia investigadora tiene en cuenta la innovación potencial para el progreso de aplicaciones de negocios o las posibilidades de mejoras significativas de los métodos, los procesos o las técnicas industriales existentes (Hicks et ál., 2000).

Podemos distinguir, así, cuatro dimensiones asociadas a las principales etapas en la creación y difusión del conocimiento, y que son susceptibles de ser evaluadas en busca de la excelencia: $a$ ) entrada (inputs): inversiones, capital humano, capital físico, infraestructura y entorno social e intelectual; $b$ ) materia procesada (throughputs): procesos que combinan las entradas, actividades e infraestructuras necesarias para alcanzar los objetivos; $c$ ) resultados (outputs): nuevos hallazgos

Scire. 12 : 2 (jul.-dic. 2006) 87-98. ISSN 1135-3716. 
científicos, nuevas técnicas científicas; $d$ ) consecuencias (outcomes): impacto de los resultados alcanzados y su influencia en la comunidad y en el entorno de la organización investigadora.

Una dimensión podría valorar las entradas (inputs) necesarias para lograr la excelencia (calificaciones académicas de los investigadores), otra se encargaría de la calidad percibida de los resultados de investigación (impacto de las citas de los artículos en revistas científicas) o del impacto económico de los resultados (éxito comercial, innovaciones tecnológicas). Ninguna de estas dimensiones es esencial por sí sola para comprender la excelencia en su totalidad. Entonces: ¿se pueden desarrollar medidas válidas para conocer los rasgos que caracterizan la calidad de las instituciones investigadoras?, ¿permiten estas medidas e indicadores la comparación entre países y disciplinas en el ámbito internacional?

\subsection{Midiendo la excelencia}

Un parámetro muy importante a tener en cuenta en la evaluación del rendimiento de la investigación es la influencia científica internacional. Desde este punto de vista, resulta esencial conocer si el rendimiento de la investigación analizada ha sido bajo, alto o, en el caso de ser excelente, muy alto. Para ello se precisan medidas que permitan detectar e identificar ese rendimiento extraordinario. La evaluación bibliométrica del rendimiento de la investigación se basa en la idea de que los científicos que tienen algo importante que decir lo hacen a través de revistas académicas internacionales. Tener el respeto y el reconocimiento de los colegas y competidores tras la realización científica es un paso importante para consolidar una reputación de excelencia.

Otro paso es conocer la influencia intelectual de los resultados obtenidos en términos de contribuciones al progreso científico y al retroceso de las fronteras de la investigación; en particular, el "impacto" dentro de la comunidad de usuarios, especialmente en los grupos de investigadores a nivel internacional. Para ello pueden usarse las referencias explícitas (citas) a documentos dentro de otras publicaciones científicas escritas por compañeros investigadores como medida de ese impacto en el entorno científico. Lograr una posición elevada en el listado global de impacto de citas dentro de una disciplina es sin duda una pieza decisiva para determinar el estatus científico de un documento y de los investigadores e instituciones asociados a él. Diversos estudios apoyan la idea de que un elevado número de citas de un artículo científico tiene correlación positiva con las opiniones de expertos sobre la importancia de ese documento, y con otros indicadores de calidad científica de las publicaciones científicas (Tijssen, Visser y Leeuwen, 2002). El recuento de documentos muy citados permite medir y comparar la excelencia científica a nivel de departamentos, laboratorios y universidades dentro de una misma área científica (Tijssen, Visser y Leeuwen, 2002; Raan, 1999).

Scire. 12 : 2 (jul.-dic. 2006) 87-98. ISSN 1135-3716. 
El desarrollo de nuevos métodos bibliométricos avanzados, que incluyen el establecimiento de nuevas metodologías e indicadores bibliométricos, ha supuesto el paso de indicadores de impacto basados en la media (impacto medio de todos los documentos publicados por una unidad) a indicadores que reflejan la presencia de los trabajos en la parte superior de las distribuciones de citas (número de documentos muy citados).

Cada tipo de indicador refleja una dimensión específica del concepto general de desarrollo científico. Consecuentemente, la aplicación de un indicador simple puede ofrecer una visión incompleta del desarrollo de una unidad. Por ello, es preciso combinar distintos tipos de indicadores para medir y localizar la excelencia con el fin de ofrecer herramientas útiles y fiables para la evaluación a los encargados de la toma de decisiones (Tijssen, 2003; Leeuwen et ál., 2003).

\subsection{Indicadores bibliométricos}

A continuación reseñaremos los indicadores bibliométricos, tanto unidimensionales como avanzados, que han sido utilizados por los principales trabajos científicos que han tratado de establecer con la menor ambigüedad posible la noción de excelencia en la investigación.

1. Total de documentos publicados por una institución (P): recuento del número de trabajos publicados por una institución durante el período de tiempo establecido para el análisis. Será necesario especificar la tipología de los documentos considerados.

2. Total de citas recibidas (C): recuento del total de citas recibidas por el conjunto de documentos de P. Será necesario especificar si se incluyen o se excluyen las autocitas.

3. Total de citas recibidas por publicación (CPP): recuento del total de citas recibidas por el conjunto de documentos de $\mathrm{P}$ dividido por el número total de documentos de P. Será necesario especificar si se incluyen o se excluyen las autocitas.

4. Porcentaje de artículos no citados durante el período (\%Pnc): porcentaje de artículos de $\mathrm{P}$ que no han recibido ninguna cita a lo largo del período de estudio seleccionado.

5. Inmediatez de cita (IC): número de citas recibidas por los artículos de P durante el mismo año de su publicación dividido por el número total de artículos publicados ese año por esa institución.

6. Índice de prioridad (IP): normalización del volumen de las publicaciones realizadas por una institución, una región o un país, para facilitar la comparación en distintos niveles. 


$$
I P=\frac{\text { producción española física / producción total española }}{\text { producción mundial física / producción mundial total }}
$$

7. Media de citas de las revistas en las que se ha publicado (JCSm): cálculo de la media de citas de las revistas en las que se han publicado los trabajos. Se tendrán en cuenta tanto el tipo documental y el año de publicación como las distintas disciplinas en las que se engloban las revistas.

8. Media de citas de los campos científicos en los que se ha publicado (FCSm): cálculo de la media de citas de las disciplinas científicas en las que se han publicado los trabajos. Se tendrán en cuenta tanto el tipo documental como el año de publicación.

Estos dos últimos indicadores se calculan a partir de los datos obtenidos de las publicaciones editadas por el Institute for Scientific Information (ISI) de Filadelfia. Cuando una revista aparezca clasificada en varios campos, el número de citas se computará de acuerdo con el número de campos asignados. Es decir, un documento clasificado en $\mathrm{N}$ campos se contará como $1 / \mathrm{N}$ en cada uno de ellos. Lo mismo ocurrirá con su FCSm.

Con el fin de establecer comparaciones nacionales e internacionales pueden calcularse dos indicadores de impacto normalizados: $a$ ) CPP/JCSm: media de citas de $\mathrm{P}$ dividida por el número medio de citas de las revistas en las que publica la institución; $b$ ) CPP/FCSm: media de citas de P dividida por el número medio de citas del campo científico en el que publica la institución.

Si el resultado en ambos casos está por encima de 1, la institución es citada con mayor frecuencia que la media de publicaciones del campo o que la media de las revistas en que publica.

1. JCSm/FCSm: si su valor se encuentra por encima de 1, el número medio de citas del conjunto de revistas en las que el centro ha publicado supera la media de citas de todos los trabajos publicados en el campo, pudiéndose concluir que la institución publica en revistas de alto impacto relativo.

2. Trabajos muy citados (HCP): según Glänzel, Rinia y Brocken (1995), los artículos muy citados (highly cited papers) representan la verdadera elite entre el total de las publicaciones producidas por un país, una región o una institución en un determinado campo. Estos artículos tienen una excelente recepción, y por tanto deben ser considerados como muy relevantes en el contexto de los análisis bibliométricos en niveles macro y meso. Los métodos desarrollados para determinar las publicaciones muy citadas son variados. Generalmente, se selecciona un número fijo de publicaciones (un número $n$ de las más importantes en un campo), un determinado cuartil de un listado de citas (los diez artículos más citados) o un umbral fijo (los documentos citados más de 400 veces). De acuerdo con la definición de Glän-

Scire. 12 : 2 (jul.-dic. 2006) 87-98. ISSN 1135-3716. 
zel y Schubert (1992), un artículo será muy citado si ha recibido al menos diez veces el número medio de citas en un campo determinado - 10·max $(1, s)$, donde $s$ es la media de citas observada (MORC)—. Es decir, un artículo será muy citado si lo ha sido al menos diez veces más que la media de artículos en ese campo. De este modo se evita que, si el número medio de referencias en un campo determinado es muy bajo, un artículo citado una o dos veces pueda ser considerado muy citado, hecho que contradice la noción de citas frecuentes.

3. Actividad de alto impacto (HIA): Glänzel y Schubert introducen este concepto para comparar el reparto relativo de artículos muy citados en un país dado frente a otros países. Este indicador, definido por analogía con el índice de actividad, será retocado para permitir la evaluación del reparto nacional en todos los artículos muy citados publicados por los países seleccionados en cada campo:

$$
H I A_{f}=\frac{\text { publicaciones nacionales en ese campo }}{\text { reparto docs. muy citados en todos los países de ese campo }}
$$

4. Índice de colaboración de alto impacto (HICA): relación entre los datos nacionales y el total de los mostrados. El HICA expresa la relación del reparto de las copublicaciones muy citadas por dos países respecto del total de artículos muy citados internacionalmente publicados por los países seleccionados, y el reparto de todas las copublicaciones de los dos países en el total de las publicaciones internacionales del conjunto de países.

$$
H I C A=\frac{\text { vínculos en todos los artículos internacionales muy citados }}{\text { reparto de colaboración por países }}
$$

5. Hot warm papers (HWP): el ISI define hot paper como aquella publicación científica publicada en los últimos dos años que en los dos últimos meses del período analizado ha resultado más citada que el resto de documentos de esa disciplina por documentos publicados en el mismo período de tiempo. El ISI considera HWP a aquellos que se encuentran en el top $0,1 \%$ de cada uno de los veintidós campos en los que divide la ciencia y que no han sido seleccionados en el bimestre anterior.

\section{Precauciones en torno a la excelencia}

La naturaleza desigual de los resultados de investigación en lo referente al recuento de citas ha sido demostrada en diversos estudios. Gran parte de los artículos científicos nunca son citados o lo son rara vez en la literatura científica posterior, mientras que unos pocos trabajos concentran el mayor número de citas (Aksnes y Siversten, 2004; Molas-Gallart y Salter, 2002; Tijssen, Visser y Leeuwen, 2002).

Scire. 12 : 2 (jul.-dic. 2006) 87-98. ISSN 1135-3716. 
Es, por tanto, preciso prestar especial atención al patrón asimétrico de la distribución de citas, que solo se revelará de forma evidente cuando se trabaje con el número de citas observadas, y nunca cuando se haga con el número de citas esperadas.

Como ya hemos visto, el problema de la política científica es cómo distribuir los fondos para la investigación en estas condiciones. Es importante darse cuenta de que es imposible saber a priori qué proyectos o programas van a tener éxito. Todos los proyectos de investigación son evaluados mediante mecanismos que intentan medir la calidad y el impacto de la investigación, pero en las primeras fases de su desarrollo estas evaluaciones son enormemente subjetivas y se basan en suposiciones.

Algunos pueden caer en la tentación de pensar que este problema se supera mediante la financiación de grupos que ya han demostrado una trayectoria de excelencia en su trabajo; que basta con apoyar a los mejores para obtener el máximo rendimiento de la inversión realizada; que, dado que los resultados de investigación son por definición inciertos, reducir al mínimo el fracaso es premiar la calidad demostrada en el pasado. En ese caso, el rendimiento excelente se convertiría, pues, en el principal criterio para dotar de fondos a la investigación, concentrando los recursos en un pequeño número de grupos que contarían con los mejores investigadores.

Sin embargo, las técnicas de investigación empleadas para detectar la excelencia son, como ya hemos dicho, comparativas. Los grupos identificados como excelentes por esas medidas lo son en virtud de su comparación con toda la población de investigadores. En este contexto comparativo, los resultados excelentes únicamente pueden ser obtenidos por unos pocos. Según esta interpretación, la excelencia es un concepto relativo que solo existe gracias a la comparación con una población mayor en la que predominan los grupos e investigadores normales. Sin normalidad no puede existir excelencia. Si los políticos, basándose en la excelencia, apuestan por ese modelo de financiación, eliminarán la cola de la distribución estadística que en un futuro quizá convierta en normales a algunos de los antaño considerados buenos investigadores.

Los responsables de las políticas científicas deben comprender, además, que la variedad y la redundancia son necesarias en la ciencia porque solo así es posible garantizar la entrada de nuevos investigadores al sistema. Esos nuevos científicos suelen situarse en las fronteras de las disciplinas, trabajando en nuevas y prometedoras líneas de investigación a largo plazo.

A todo ello hay que unir el hecho de que la excelencia no es permanente. Aquellos que obtienen rendimientos medios en un momento dado pueden lograr una excelente investigación en el futuro; y viceversa, grupos de investigación excelentes 
pueden quedarse obsoletos o resultar inoperantes, especialmente si su acceso a los fondos resulta cada vez más fácil.

\section{Conclusiones}

Los indicadores bibliométricos miden con gran precisión los logros y la trayectoria científica de determinadas disciplinas, especialmente de aquellas relacionadas con la investigación básica. Estos indicadores son los únicos basados en datos empíricos que pueden obtenerse en la actualidad, y son válidos en el primer estadio de la búsqueda de la excelencia de la investigación. El uso de indicadores de impacto de citas ofrecerá información más explícita y objetiva sobre el impacto externo y la utilidad del conocimiento científico producido.

Sin embargo, este tipo de estudios basados en indicadores bibliométricos requiere de unos datos estadísticos fiables y suficientemente informativos. En el caso de estudios con pocos sujetos a menudo los datos no son lo suficientemente precisos para extraer conclusiones o establecer comparaciones. Además, la obtención de los datos, y por tanto la calidad de los resultados, suele estar supeditada a la fuente de información empleada.

Debemos tener también presente que el uso de indicadores de citas agregados para evaluar el desarrollo científico ha de incluir el análisis de las distribuciones de citas resultantes, puesto que habitualmente muestran una alarmante asimetría. Un análisis de los datos subyacentes de estos indicadores permitirá, además, detectar trabajos de gran influencia. El estudio basado en citas tiene que ser capaz de localizar las desviaciones de la ciencia normal, aquella que nunca o casi nunca es citada.

Por otro lado, las autoridades públicas, los rectores, los directores de institutos y centros de investigación y los directivos de empresas dedicadas a investigación encuentran cada vez más dificultades para equilibrar la balanza de la mejora de los desarrollos y la reducción de costes. La búsqueda de recursos que mejoren el coste-efectividad de los gastos derivados de la investigación se ha convertido para ellos en algo prioritario, puesto que es la única forma de asegurar la viabilidad futura de la misma. Resulta, pues, de vital importancia la obtención de información que permita la comparación con el fin de poder tomar las mejores decisiones debidamente justificadas.

En definitiva, el problema al que se enfrentan los gestores y administradores es determinar a priori qué será investigación de excelencia. Pero la certidumbre en la generación de resultados científicos deja paso a la suerte y a la casualidad, haciendo muy difícil conocer cuándo y dónde surgirá la excelencia.

Ya hemos visto que la excelencia científica es polifacética. Los criterios y medidas hasta ahora empleados para detectar la excelencia de las instituciones y los

Scire. 12 : 2 (jul.-dic. 2006) 87-98. ISSN 1135-3716. 
grupos de investigación se basan en el historial de resultados pasados. Tomar exclusivamente en consideración estas medidas comparativas para establecer propuestas o articular acciones políticas orientadas a evaluar o promover los centros de excelencia resulta, en el mejor de los casos, cuestionable.

\section{Notas}

(1) Este trabajo se ha realizado gracias a la financiación obtenida en la convocatoria de 2004 de proyectos de investigación en humanidades, ciencias sociales y económicas de la Comunidad de Madrid (Orden 1452/2004, de 16 de abril).

\section{Referencias}

Aksnes, D. W.; Sivertsen, G. (2004). The effect of highly cited papers on national citation indicators. // Scientometrics. 59:2 (2004) 213-224.

Glänzel, W.; Rinia, E. J.; Brocken, M. G. M. (1995). Highly cited papers: a bibliometric study of highly cited European physics papers in the 80s. // Research Evaluation. 5:2 (1995) 113-122.

Glänzel, W.; Schubert, A. (1992). Some facts and figures on highly cited papers in the sciences, 1981-1985. // Scientometrics. 25 (1992) 373-380.

Hicks, D.; Breitzman, A.; Halmilton, K.; Narin, F. (2000). Research excellence and patented innovation. // Science and Public Policy. 27:5 (2000) 310-320.

Molas-Gallart, J.; Salter, A. (2002). Diversidad y excelencia: consideraciones sobre política científica. // The IPTS Report. 66 (jul. 2002).

Leeuwen, T. N. van; Visser, M. S.; Moed, H. F.; Nederhof, A. J.; Raan, A. F. J. van (2003). The Holy Grail of science policy: exploring and combining bibliometric tools in search of scientific excellence. // Scientometrics. 57:2 (2003) 257-280.

Raan, A. F. J. van (1999). Evaluación de la excelencia científica de programas de investigación: un punto primordial en la toma de decisiones. // The IPTS Report. 40 (dic. 1999).

Rovira, L. (2003). Mapa de la excelencia en física y química de las universidades españolas. Gerona: Universidad.

Tijssen, R. J. W. (2003). Scoreboards of research excellence. // Research Evaluation. 12:2 (2003) 91-103.

Tijssen, R. J. W; Visser, M. S.; Leeuwen, T. N. Van (2002). Benchmarking international scientific excellence: are highly cited research papers an appropriate frame of reference? // Scientometrics. 54:3 (2002) 381-397. 